larities between $\mathrm{HCO}$ and $\mathrm{HNO}$ in all probability do not extend as far as the formation mechanisms. The formation of $\mathrm{HCO}$ is likely to depend on the availability of ionised carbon in moderate-density clouds. Ionised nitrogen may not be so readily available, so that the best suggestions for HNO formation come from Dalgarno

$$
\mathrm{NH}_{s}^{+}+\mathrm{O} \rightarrow \mathrm{H}_{2} \mathrm{NO}^{+}+\mathrm{H}
$$

followed by

$$
\mathrm{H}_{2} \mathrm{NO}^{+}+e \rightarrow \mathrm{HNO}+\mathrm{H}
$$

the initial $\mathrm{NH}_{s}^{+}$being derivable either from neutral nitrogen

$$
\mathbf{N}+\mathbf{H}_{3}^{+} \rightarrow \mathbf{N H}_{3}^{+}
$$

or from the ion, $\mathrm{N}^{+}$, and unionised hydrogen molecules.

The letter in the Astrophysical Journal also reports a new unidentified line at a frequency of $81,505 \mathrm{MHz}$ which may be a weak maser and observations of previously detected transitions of ketene $\left(\mathrm{H}_{2} \mathrm{CCO}\right)$, dimethyl ether $\left(\mathrm{CH}_{3} \mathrm{OCH}_{3}\right)$ and cyanoacetylene (HCCCN). A further unidentified line previously reported by Turner (Astrophys. J. Lett. 213, L75; 1977) was also observed and ascribed to thioformaldehyde $\left(\mathrm{H}_{2}\right.$ C.S $)$ or cyanoacetylene $\left(\mathrm{HC}_{2}{ }^{13} \mathrm{CN}\right)$.

The ambiguous identification, the unidentified line and above all the detection of an interstellar NO bond will all provide spurs for theoreticians to perform accurate calculations, spectroscopists to measure frequencies as well as encouragement for radioastronomers to confirm and extend this study, perhaps once more searching for nitric oxide in interstellar regions.

\section{Hydrothermal waters and heat flow}

\section{from Peter J. Smith}

THE heat flowing outwards through the oceanic crust reaches the Earth's surface in three ways-by conduction, convection and radiation from the mantle and core, by conduction from radioactive sources in the crust itself, and by direct transport in the hot mantle material which rises at active oceanic ridges to form new lithosphere. Of the three, the most important is the last; indeed, more than $50 \%$ of the Earth's heat loss (that is, from oceanic and continental regions combined) takes place at oceanic ridges alone. With the possible exception of the few

\title{
Speed of light and relativity
}

\section{from P. E. Hodgson}

EINSTEIN's special theory of relativity is based on two postulates-first that the laws of physics that hold in one coordinate frame also hold in any other coordinate frame moving with uniform velocity with respect to it, and second that the speed of light is independent of the velocity of the source. From these two postulates the theory of relativity can be derived.

The second of these two postulates can be tested experimentally and this thas already been done in several ways. Among the most accurate is the study of gamma rays emitted from the decay of relativistic uncharged pions produced in very energetic nucleonnucleon collisions. In one of these measurements the pions had an energy of $6 \mathrm{GeV}$ and thus a velocity very nearly that of light, so that if the velocity of the source is added to that of the light, the gamma rays from their decay would have nearly twice the velocity of light, whereas if the velocities do not add the velocity is just $c$. There is thus nearly a factor of two between the velocities expected on the two possibilities, and this can be measured accurately by a time-of-flight method. If the result is expressed by saying that the measured velocity is

$$
c^{\prime}=c+k v
$$

where $k$ is a constant and $v$ the velocity of the source, then the two possibilities correspond to $\mathrm{k}=0$ and $\mathbf{k}=1$. The measurements showed that $\mathbf{k}$ is smaller than $10^{-4}$, which confirms the second postulate to high accuracy.

It is, however, always interesting to see if such an important result can be checked to even higher accuracy, and this has recently been done by Kenneth Brecher of the Center for Space Research at Massachusetts Institute of Technology (Phys. Rev. Lett. 39, 1051; 1977). He made use of the regularly pulsing $X$-ray sources in binary star systems. Since in a binary star one component is orbiting round the other, if the system is observed in the plane of the orbit, for part of the period one star appears to be moving towards us and the other away from us, and the opposite happens half a

local continental areas of high heat flow capable of providing geothermal power, oceanic ridges are therefore the most interesting of all the Earth's thermal features. They are also the most important in the sense that study of their thermal characteristics is capable in principle of throwing light on period later. If the velocity of the source is added to that of the light then we would not see two stars rotating around each other but instead would see complicated effects depending on the distance of the stars. In particular we would not see them continuously: they would appear and disappear as the various beams from the stars in different positions overtake one another as a result of their different velocities.

This method of confirming that the velocity of light is independent of that of the source was suggested long ago, but for light in the visible region it suffers from the objection that the radiation from a distant star is scattered as it goes through the intervening interstellar matter, and is then replaced by reradiated fields produced by the dipoles of the medium. This wave is then propagated with the phase velocity of the medium. Calculations show that for light in the visible region the characteristic length for the extinction of the original radiation is about two light years, which is less than the distance of the nearest star.

This objection does not apply to the $70 \mathrm{keV} X$ rays emitted from some binary star systems, as they are much more penetrating and the interstellar extinction is negligible. Such stars thus provide a very sensitive way of testing the second postulate.

Brecher applied this method to three binary X-ray sources, and determined their distances, orbital periods, and Doppler velocities. A small but finite value of the constant $k$ would affect the eclipse times and the apparent eccentricity of the orbit, and so measurements of these quantities enable upper limits to be set to k. A careful analysis of the results of the measurements showed that for one of the stars $k$ is less than $2 \times 10^{-8}$, and for the other that it is less than $4 \times 10^{-10}$. These limits are much less than those set by the pion method. The second postulate is thus established to much greater accuracy than ever before.

P. E. Hodgson is a lecturer in Nuclear Physics in the University of Oxford.

some of the details of the plate tectonic processes which brought the heat to the surface in the first place.

Unfortunately, however, oceanic ridges are precisely those regions where heat flow measurement is most difficult and the results most uncertain. Conventional heat flow probes require for 